\title{
A propos du fauchage ou basculement des têtes de couches
}

P. HABIB ET P. DUFFAUT

Lorsque la stratification ou la schistosité est parallèle au versant et très redressée, on observe souvent sur une pente naturelle que le haut des couches est déversé vers l'aval (Fig. 1a). La déformation correspondante peut affecter des profondeurs diverses. Mais comme les diversités des constitutions minéralogiques ou géornécaniques des massif́s sont très grandes 甘i est assez probable que des mécanismes diffẻrents peuvent engendrer des effets analogues sur des massifs différents. Depuis le fluage du sol (Fig. 1b) jusqu'á l'effondrement des livres sur une étagère (Fig. 1c) en passant par les basculements des têtes de couches observés par les géologues il est bien clair que les bases physiques de ces phénomènes doivent correspondre à des comportements mécaniques qui ne sont pas comparables.

Pour que puisse se produire, ta « poussée

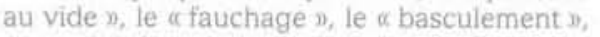
en anglais le toppling, puisque ces dénominations ont été utilisées pour décrire ces phénomènes, il faut cependant que diffërents facteurs géométriques communs existent au préalable.

Il faut d'abord que l'érosion ait créé une pente suffisante. Il faut ensuite une stratification verticale ou subverticale, éventuellement avec un léger surplomb vers l'aval. Il faut, enfin, qu'au pied de la pente il y ait eu, à un moment donné, suffisamment de place pour que les matériaux situés immédiatement à lamont puissent venir s'y loger créant ainsí une nouvelle liberté de mouvement qui permet à un phénomène régressif de se développer. C'est par exemple ce qui se produit souvent dans le cas du façonnage du lit glaciaire en $U$ au sein d'une vallée en $\mathrm{V}$. Le pied de la pente est coupé et produit une rupture de pente. Il y a un degré de líberté vers le vide que la pesanteur va exploiter.

Le terme " poussêe au vide n, utilisé parfois pour expliquer cette transformation est. certes, très imagé mais il n'explique rien même s'il sous-entend bien l'existence d'un vide préalable. Le moteur du mouvement est fondamentalement la pesanteur, que ce soit le poids des strates et leur déséquilibre ou la pression de l'eau dans les fissures entre les strates lou la pression de courant s'il existe des différences de perméabilité entre des couches adjacentes). Mais en montagne la dilatation de l'eau interstitielle provoquée par le gel est aussi un moteur capable de développer des efforts considérables et même si les déplacements sont petits, la répétition saisonnière suffit pour bousculer progressivement toute la structure,

La description d'un mode possible de mouvement présentée ici ne semble pas avoir été signalée jusqu'à présent. Ce mouvement a besoin lui aussi des mécanismes géométriques ou hydrauliques, cités ci-dessus. Examinons donc le mouvement des strates verticales de la figure 2. Une fois basculé, l'empilement de strates garde la mème épaisseur. On peut le
NDLE: Les discussions sur cét article sont acceptées jusqu'au $1^{\text {te }}$ septembre 2003.

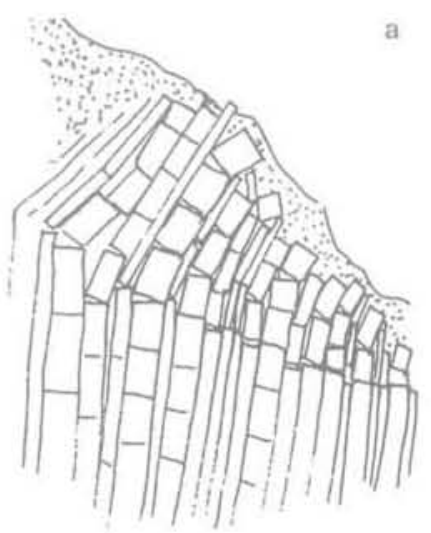

a
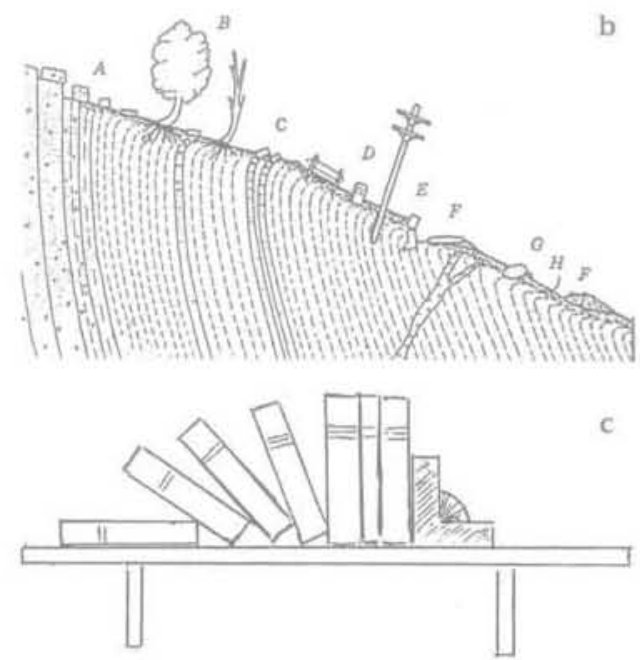

FiG.1 (a) Basculement de blocs (d'après Hoek et Bray, 1976, cité par MerrienSoukachoff et al., 2001).

(b) Exemples de fluage du sol (d'après Sharpe, 1938, cité par Taylor, 1948).

(c) Effondrement d'une rangée de livres sur une étagère. 


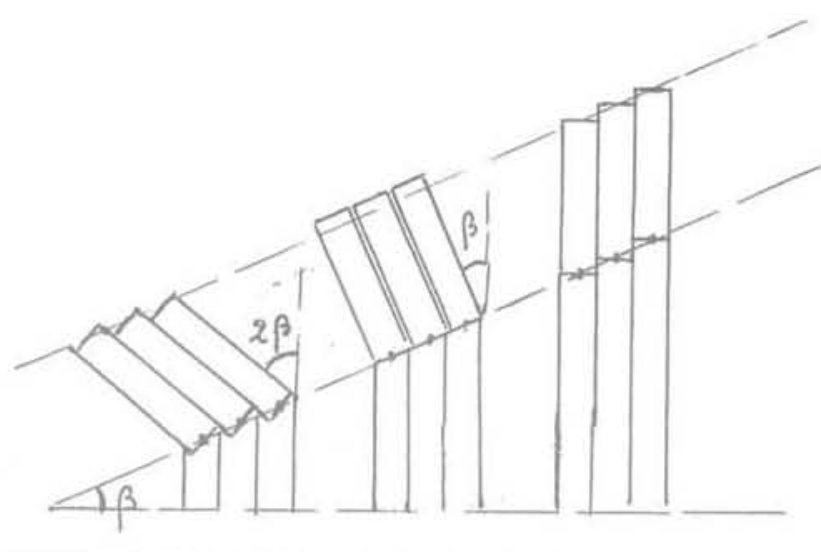

FG. 2 Rotation des éléments de strates.

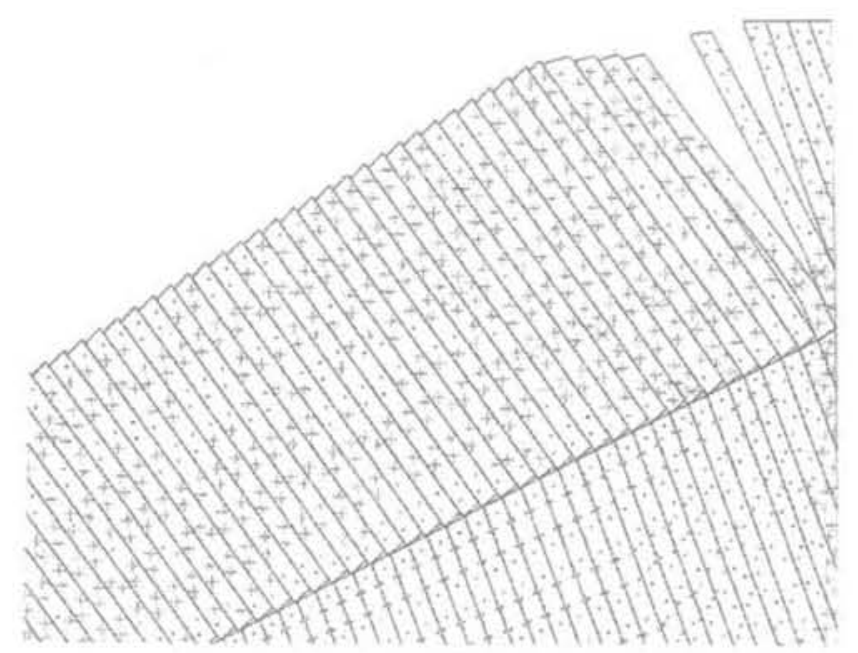

Fig.3 Modèle d'éléments distincts basculés d'après Merrien-Soukatchoff et al., 2001. voir sur des modèles géomécaniques par èléments díscrets comme celui de la figure 3 . Et c'est la grande différence par rapport aux livres sur un rayon de bibliothèque de la figure 1c où les intervalles à leurs pieds sont d'autant plus grands que les livres sont couchés. Sur la figure 2, on présente la rotation de trois éléments de strate. On peut admettre en première approximation que la ligne des points d'articulation est parallèle à la ligne de pente du talus d'inclinaison $\beta$. Le début du mouvement provoque un desserrement des couches qui est maximal lorsque les éléments sont perpendiculaires à la ligne de pente (c'est-à-dire lorsqu'ils ont tourné d'un angle $\beta$ ).

Si e est l'épaisseur constante des strates, l'intervalle entre elle est: $e \frac{1-\cos \beta}{\cos \beta}$. Si la rotation continue les couches se resserrent et après une nouvelle rotation de $\beta$, elles reviennent au contact et elles ne peuvent plus aller plus loin (Fig. 2). S'il y a de I'altération entre les couches et un peu de compressibilité la rotation maximale peut être un peu supérieure à $2 \beta$. Si l'intervalle entre les couches a été un peu rempli par des débris au cours du passage au voisinage à la normale à la pente, la rotation maximale peut être inférieure à $2 \beta$. Ultérieurement le phénomène peut se reproduire à un niveau plus profond et donner le mécanisme de la figure 4 où les morceaux de la première couche doivent glisser les uns sur les autres et donnent un dessin en crochets, typique des observations de Lugeon et Oulianoff (1922) ou de Bordet (1959), Lorsque les couches sont minces elles sont plus fragiles ou plus flexibles et elles peuvent donner une allure plus continue que celle qui est dessinée ici.

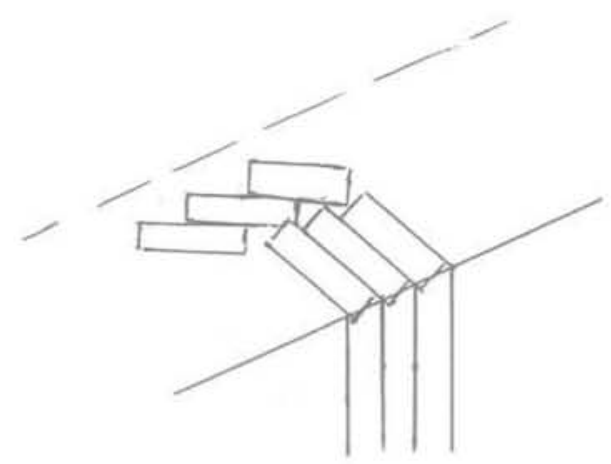

fiG.4 Mécanisme profond.

\section{$\overline{\text { Bibliographie }}$}

Alfonsi P., Durville J.L., Rachez X. "Quelques applications de la méthode des éléments distincts en mécanique des roches . Bulletin des $L P C, \mathrm{n}^{\circ} 214,1998, \mathrm{p} .36$.

Bordet $\mathrm{C}$. - - Les modes de circulations de l'eau dans les massifs cristallins $y$. Congrès et colloques de I'Université de Liège, vol. 14, 1959 , p. 65.
Hoek E., Bray J.W. - « Rock Slope Engineering ${ }^{\prime}$. The Institution of Mining and Metallurgy, 1981

Goodman R., Bray J.W. - Toppling of Rock Slopes ». Conf. on Rock Eng. for Foundations and Slopes, Boulder, ASCE, vol. 2, 1976.

Lugeon M., Oulianoff N. - - Sur le balan- cement superficiel des couches ग. Bull. Soc. vaudoise des sciences naturelles, vol. $54, n^{\circ} 206,1922$.

Merrien-Soukatchoff $V_{\text {., }}$ Quenot $X$. Gugliemi Y. - « Modélisation par éléments distincts du phénomène de fauchage gravitaire n. Revue française de géotechnique, $n^{\circ}$ 95-96, 2001, p. 137. 